\title{
Ergoespirometria em atletas paraolímpicos brasileiros
}

\author{
Antônio Carlos da Silva ${ }^{1,2}$ e Fernando Carmelo Torres ${ }^{1,2}$
}

\section{RESUMO}

A partir dos testes de aptidão física realizados em atletas paraolímpicos brasileiros, na preparação para as Paraolimpíadas de Sidney 2000, este artigo objetiva discorrer sobre a utilização da Ergoespirometria como um importante método de avaliação funcional, cujas aplicações podem beneficiar desde portadores de patologias até os atletas de alto nível, incluindo-se os portadores de deficiências físicas ou mentais. São mostrados os resultados dos principais parâmetros analisados (consumo máximo de oxigênio - $\mathrm{V}_{2} \max$ - e limiares anaeróbios ventilatórios) nestas avaliações com atletas paraolímpicos, de diversas modalidades e com diferentes tipos de deficiências, além de suas bases fisiológicas gerais, a metodologia e protocolos empregados. Salientam-se também certas características especiais que estes testes apresentam, principalmente em portadores de deficiências, como especificidade, cuidados de segurança, processos de motivação e entendimento. Finalmente, a pouca disponibilidade de trabalhos deste tipo publicados na literatura, especialmente com portadores de deficiências brasileiros, torna necessária a divulgação de estudos nesta linha de pesquisa. Estes trabalhos certamente poderão auxiliar para um melhor conhecimento sobre este grande contingente de portadores de deficiências que temos no Brasil e no mundo, colaborando para a implementação de diretrizes e ações mais efetivas voltadas especificamente para estes indivíduos, não apenas no âmbito competitivo,

1. Disciplina de Neurofisiologia e Fisiologia Endócrina da Universidade Federal de São Paulo/Escola Paulista de Medicina.

2. Laboratório de Fisiologia Respiratória e do Exercício (Lafirex) do Centro de Estudos de Fisiologia do Exercício (CEFE) da Universidade Federal de São Paulo/Escola Paulista de Medicina.

Submetido em: 4/4/02

Versão revisada recebida em: 1/5/02

Aceito em: 19/5/02

Endereço para correspondência:

Dr. Antonio Carlos da Silva

CEFE - Centro de Estudos de Fisiologia do Exercício

Rua Botucatu, 862, 5 andar

04023-062 - São Paulo, SP

E-mail: anton@ecb.epm.br mas para qualquer pessoa portadora de necessidades especiais.

Palavras-chave: Ergoespirometria. Consumo máximo de oxigênio. Limiar anaeróbio. Atleta paraolímpico. Atleta portador de deficiência.

\section{ABSTRACT}

\section{Ergometric evaluation in Brazilian paralympic athletes}

The purpose of the present article is to discuss the use of incremental exercise test as an important method of functional evaluation of disabled individuals, based on the presentation of the results of the Brazilian athletes that participated in the Sydney Paralympic Games. In addition to the presentation of the analyzed parameters (maximal oxygen uptake - $\dot{V}_{2}$ max - and ventilatory anaerobic threshold) for athletes from various sports and types of disability, the physiological basis and methodology used are described. Certain characteristics pertaining to disabled athletes, such as specificity, safeguards, understanding and motivation are explained in the text. Finally, the publication of data regarding this specific group will help the implementation of more effective actions, not only in competitive sports, but also for the rehabilitation of the great contingent of persons with physical and/or mental disabilities in Brazil.

Key words: Ergometry. Oxygen uptake. Anaerobic threshold. Disability. Athlete.

\section{INTRODUÇÃO}

Os avanços científicos e tecnológicos, aliados à gradativa queda do custo de alguns equipamentos, têm levado a um aumento do número de estações de Ergoespirometria, antes restritas quase que exclusivamente aos grandes centros de pesquisa universitários. Por outro lado, um relativo desconhecimento, por parte de alguns profissionais, sobre as várias e importantes informações que este teste pode proporcionar tem, em parte, subestimado sua aplicação prática, dificultando que esta expansão aconteça num ritmo maior.

Neste artigo, vamos discutir os conceitos fisiológicos básicos envolvidos na Ergoespirometria, as metodologias 
empregadas e as suas principais aplicações, bem como mostrar protocolos e resultados encontrados em portadores de deficiências, tendo por base atletas brasileiros que representaram o país nas Paraolimpíadas de Sidney 2000.

\section{Bases fisiológicas}

A mensuração das trocas gasosas respiratórias se faz pela determinação dos volumes de oxigênio e de gás carbônico da ventilação pulmonar, num período de tempo preestabelecido. Atualmente, a técnica mais utilizada para isto é a da "espirometria de circuito aberto" computadorizada, onde o avaliado inspira o ar ambiente e tem sua expiração direcionada para um equipamento com analisadores eletrônicos de gases. Através da mensuração da ventilação pulmonar (por fluxômetro) e sabendo-se as frações de $\mathrm{O}_{2}$ e de $\mathrm{CO}_{2}$ inspirados (20,93 e 0,03\%, respectivamente), as diferenças com as medidas obtidas no ar expirado possibilitam a informação da quantidade de $\mathrm{O}_{2}$ consumido e de $\mathrm{CO}_{2}$ produzido.

Consumo máximo de oxigênio ( $\left.\dot{\mathrm{V}}_{2} \max \right)$ - pode ser definido como a máxima capacidade de captação (pulmões), transporte (coração e vasos) e utilização do oxigênio (principalmente pelos músculos), durante exercício dinâmico, envolvendo grande massa muscular corporal ${ }^{1}$. É representado pela abreviatura " $\mathrm{V}_{2}$ max", onde $\mathrm{V}$ é o volume consumido por minuto. A determinação do $\dot{\mathrm{V}}_{2}$ max tem sido amplamente utilizada na literatura como um dos principais indicadores da capacidade para realização de exercícios físicos de longa duração ${ }^{1}$. Como o consumo de oxigênio cresce proporcionalmente com a carga de trabalho ${ }^{2} \mathrm{e}$, sendo o $\dot{\mathrm{V}} \mathrm{O}_{2}$ max o "limite" deste aumento, existe uma relação direta entre este parâmetro e a capacidade de desempenho para atividades com duração acima de 2 a 3 minutos, quando a produção energética aeróbia passa a predominar ${ }^{1}$. Entretanto, não podemos esquecer que, no esforço máximo, a produção energética total é determinada pela produção aeróbia de energia, traduzida pelo $\dot{\mathrm{V}}_{2} \max$, e também por um componente anaeróbio variável, cuja participação pode ser importante.

A mensuração do $\dot{\mathrm{V}}_{2}$ max pode ser indicada por várias razões, desde a avaliação da função cardiorrespiratória, em pneumo ou cardiopatas, por exemplo, até a predição de desempenho em atletas. Há diversos testes, tabelas e equações que estimam o $\dot{\mathrm{V}}_{2}$ sob condições padronizadas, numa tentativa de minimizar custos e/ou simplificar procedimentos. No entanto, em muitos casos, como em estudos científicos ou nas avaliações que exijam maior precisão (para alguns tipos de diagnóstico clínico ou de desempenho atlético), não se deve preterir a sua obtenção direta. A medida do $\mathrm{VO}_{2}$ max pela Ergoespirometria é um processo bastante confiável (coeficiente de correlação teste-reteste $=0,95$ e de erro $=2,4 \%^{3}$ ).

Num teste de esforço progressivo máximo, o aumento do $\mathrm{V}_{2}$ com a intensidade do exercício pode apresentar uma estabilização (platô), apesar de um aumento na carga de trabalho. Nesta situação, considera-se que foi alcançado um limite fisiológico do sistema de fornecimento e utilização de oxigênio, ou seja, o $\dot{\mathrm{V}}_{2}$ max. A carga de exercício pode continuar a aumentar, sem crescimento adicional do $\dot{\mathrm{VO}}_{2}$, mas isto está intimamente correlacionado à participação do metabolismo anaeróbio, dependendo também do nível de motivação do avaliado. Normalmente, é o avaliado quem interrompe o teste, após atingir uma possível "exaustão". Entretanto, por ser subjetiva, esta decisão acaba sendo muito influenciada por diversos fatores, especialmente psicológicos, que podem não levar a um esforço máximo efetivo. A observação deste platô nem sempre é possível em adultos, especialmente idosos, sedentários e doentes, além de ser raramente encontrado em crianças ${ }^{4}$. Para corroborar, também são comumente adotados como indicadores do $\dot{\mathrm{V}}_{2} \max$, embora menos precisos: uma razão de trocas respiratórias (“R”) maior que 1,0 (podendo superar 1,10 ou 1,15); a obtenção de uma FC próxima ou acima da máxima (FCmax) prevista para a idade; sinais de intolerância aos esforços (sensação subjetiva de fadiga, tontura, palidez fascial, etc.) e níveis sanguíneos de lactato atingindo ou superando 70 a $80 \mathrm{mg} / 100 \mathrm{~mL}$ de sangue (cerca de 8 a $10 \mathrm{mmol})^{1,5,6}$. Deve-se ressaltar que não é esperada a ocorrência simultânea destes critérios num mesmo teste. Procurando-se evitar a coleta de sangue, alguns cientistas 7,8 estabeleceram que a obtenção de pelo menos dois dos seguintes critérios validaria o $\mathrm{V}_{2}$ max: $\mathrm{R} \geq 1,15$; FCmax atingida dentro de um desvio de \pm 10 da FCmax prevista; e a observação de um platô de estabilização, após incremento de carga. Quando os critérios para determinação do $\mathrm{V}_{2} \max$ não são satisfeitos, ou a limitação do teste possa ter sido provocada por fatores musculares locais (e não por mecanismos hemodinâmicos centrais), utiliza-se o termo "pico de $\dot{\mathrm{VO}}_{2}$ " para o valor mais alto de consumo de oxigênio obtido durante este teste de esforço.

Os valores de $\dot{\mathrm{V}}_{2} \max$ nos homens ficam geralmente $15 \mathrm{a}$ $30 \%$ acima dos escores das mulheres ${ }^{9}$. Mesmo para atletas treinados, essa diferença pode oscilar entre 15 e $20 \%^{10}$. As discrepâncias tornam-se ainda muito maiores se adotarmos o valor absoluto $(\mathrm{L} / \mathrm{min})$ ao invés do relativo $(\mathrm{mL} / \mathrm{min} / \mathrm{kg}$ de massa corporal). Esta aparente diferença sexual tem sido atribuída, principalmente, à menor concentração de hemoglobina e maior porcentagem de gordura corporal das mulheres. O homem, em geral, pode gerar mais energia aeróbia porque possui uma massa muscular maior ("massa metabolicamente ativa"), menos gordura corporal total 
("massa inativa") e mais células carreadoras de oxigênio, para um mesmo volume sanguíneo.

Os valores de $\dot{\mathrm{V}}_{2} \max$ "absoluto" são mais comumente expressos em litros/min (L/min) ou mililitros/min (mL/ min). Normalmente, o vंO ${ }_{2} \max$ "relativo" é o mais adequado para comparações, representando a razão entre os valores absolutos e o peso corporal (geralmente em $\mathrm{mL} / \mathrm{min} /$ $\mathrm{kg}$ ). Entretanto, especialmente nos casos de pessoas com atrofia ou ausência de membro, a interpretação de resultados de consumo de oxigênio em relação ao peso corporal deve ser feita com muita cautela, pois nestas situações pode haver superestimação de valores.

Observa-se pouca ou menor evolução do $\dot{\mathrm{V}}_{2}$ max quando este é medido num tipo de ergômetro que exija grupos musculares ou movimentos diferentes daqueles utilizados no treinamento ou na prática esportiva, com os resultados mais significativos sendo demonstrados em situações similares às desempenhadas rotineiramente pelo avaliado ${ }^{11}$. Em especial, pessoas com paraplegia, amputações ou lesões de membros inferiores podem ter sua avaliação feita pela ergometria de braços. Neste caso, como salientado anteriormente, as limitações inerentes a este teste (pela menor massa muscular envolvida) fazem com que o maior valor de $\dot{\mathrm{V}}_{2}$, obtido nesta situação, seja denominado como "pico de $\mathrm{V}_{2}$ ", com os resultados sendo cerca de 20 a $30 \%$ menores caso pudessem ser realizados com as pernas ${ }^{12}$.

Alguns laboratórios têm se esforçado em disponibilizar para as pessoas com deficiência, principalmente as praticantes de modalidades esportivas, meios de execução de testes mais específicos. Para cadeirantes, por exemplo, foi proposta uma adaptação das rodas da cadeira a dispositivos ergométricos que regulam a resistência no giro das mesmas $^{13}$. Nosso laboratório (CEFE-Unifesp), que tem dedicado uma atenção especial à avaliação e estudo de pessoas com diferentes deficiências, dispõe de um ergômetro de braços e de uma esteira rolante especial, com dimensões adequadas para a colocação da própria cadeira do avaliado. Esta é presa à esteira por resistentes tiras elásticas de segurança sem, contudo, restringir a sua movimentação durante o teste. Este sistema foi implantado com sucesso visando as avaliações de atletas paraolímpicos brasileiros para "Atlanta 96".

Por fim, o $\dot{\mathrm{O}}_{2} \max$ tem uma importante influência genética. Estudos iniciais estimaram que a herança genética era responsável por até $93 \%$ da variabilidade do $\mathrm{V}_{2}$ max. Posteriormente, outros trabalhos tentaram mostrar o efeito da hereditariedade sobre $\mathrm{o} \mathrm{V}_{2} \max$, porém com a detecção de menores $\operatorname{taxas}^{14}$ (25 a 50\%). Esta herança faz com que, após alcançado o potencial geneticamente determinado, o treinamento não surta mais efeito sobre o $\dot{\mathrm{V}}_{2} \max$. Mesmo neste caso, é comum verificarem-se melhoras de desempe- nho, pois a capacidade para o trabalho prolongado também depende da tolerância a intensidades submáximas de esforço, num percentual elevado em relação ao $\dot{\mathrm{V}}_{2}$ max. O fato de atletas muito bem condicionados conseguirem prolongar o esforço, em intensidades próximas de seu $\dot{\mathrm{V}}_{2} \max$, podendo independer da melhora deste último, é um exemplo de que $\mathrm{o} \dot{\mathrm{V}}_{2} \max$ não pode justificar totalmente a capacidade de manutenção de um exercício. Tudo isto serviu para impulsionar o aparecimento de diversas linhas de pesquisa, para que se encontrassem métodos e parâmetros mais sensíveis na detecção dos efeitos do treinamento e de alterações de desempenho em cargas submáximas. Dentre estes parâmetros está o "limiar anaeróbio", discutido a seguir.

Limiares anaeróbios - desde o início dos anos 30, aceita-se que o $\dot{\mathrm{VO}}_{2}$ aumenta linearmente com o incremento de carga, mas a concentração de lactato no sangue altera-se muito pouco desde o repouso até determinadas cargas de trabalho ( 40 a $70 \%$ do $\dot{\mathrm{V}}_{2} \max$ ), durante um exercício de intensidades progressivas. Três décadas depois, surgiram protocolos de avaliação para tentar identificar o momento da transição entre as cargas onde haveria predominância do metabolismo aeróbio para aquelas em que o metabolismo anaeróbio seria o mais importante. Ultrapassado este "limite" (limiar), a concentração de lactato aumentaria, com a ventilação $(\mathrm{VE})$ e a produção de $\mathrm{CO}_{2}\left(\mathrm{VCO}_{2}\right)$ crescendo desproporcionalmente ao incremento do $\dot{\mathrm{VO}}_{2}$. Isto levou cientistas à utilização de curvas ventilatórias e de lactato para a determinação destes limiares. A partir dos anos 70, avaliações destas curvas passaram a ser adotadas com mais freqüência em exames de cardiopatas e portadores de insuficiência cardíaca ${ }^{5}$. Atualmente, a aplicação destes limiares tem sido difundida na avaliação e controle de atletas, indivíduos saudáveis ou pacientes participantes de programas de treinamento; no diagnóstico diferencial das limitações da capacidade física; e na análise do efeito de diversas terapias.

Wasserman e colaboradores ${ }^{15}$ propuseram que, nas cargas de trabalho onde ocorre aumento da concentração de lactato sanguíneo (geralmente entre 40 e $70 \%$ do $\dot{\mathrm{V}}_{2} \max$ ), estaria havendo a ativação da glicólise anaeróbia para suplementar a produção energética aeróbia. No entanto, outros autores ${ }^{16-19}$ têm defendido em seus estudos que a glicólise anaeróbia somente passaria a contribuir significativamente em cargas acima de $70 \%$ do $\dot{\mathrm{V}}_{2} \max$, propondo que se evite a correlação direta entre a aceleração da atividade do metabolismo anaeróbio e o aumento do lactato no sangue, inclusive na denominação dos limiares, sugerindo o termo "limiar de lactato" como mais adequado. A hipóxia muscular foi considerada, nos estudos iniciais, como a causa deste acúmulo ${ }^{20}$. Embora ainda seja 
um tema controverso, com alguns pesquisadores ${ }^{21,22}$ acreditando que a disponibilidade de oxigênio para a musculatura esquelética seja o fator mais importante para o acúmulo de lactato, outros trabalhos ${ }^{23-25}$ têm questionado esta tese. Uma velocidade de glicólise aumentada, com incremento da produção de NADH, pode superar a capacidade de envio dos hidrogênios $\left(\mathrm{H}^{+}\right)$para o interior das mitocôndrias $^{26}$. Esta falha de sincronia entre a formação de NADH e o sistema de transporte de hidrogênio pode causar a produção de ácido láctico (resultado do recebimento, pelo ácido pirúvico, destes hidrogênios não transportados), sem ter havido, necessariamente, hipóxia da célula muscular. Outra razão aventada é o recrutamento cada vez maior de fibras musculares "rápidas" (tipo II), pelo aumento da intensidade de esforço. Nelas predomina a transformação de piruvato em lactato, o que provoca maior produção deste último, mesmo com uma adequada oferta de oxigênio. Também é apresentada como possível causa do "limiar de lactato" uma redução da sua taxa de remoção sanguínea. Alguns trabalhos com animais sugerem que um aumento de ácido láctico pode ocorrer por uma maior produção e/ou pela diminuição de sua velocidade de remoção ${ }^{18}$. Independente destas discussões (campo aberto para a busca de conclusões definitivas), o resultado final será um incremento do lactato sanguíneo, que pode ser devido a um destes fatores ou a uma combinação deles.

Por isso, o "limiar anaeróbio" (ou "limiar de lactato") está intimamente relacionado ao ácido láctico, que apresenta variações de concentração de acordo com a intensidade de exercício, com tendência de acúmulo em cargas mais elevadas. O termo "limiar anaeróbio" é utilizado para identificar, em testes de esforço, os "limites" de intensidade onde ocorrem incrementos abruptos da concentração de ácido láctico (e, como veremos, de lactato sanguíneo), o que traz importantes repercussões fisiológicas. Neste texto, discorreremos sobre a determinação do "limiar" pelo método ventilatório, através da Ergoespirometria ${ }^{27}$. Antes, tendo em vista o papel do ácido láctico dentro do metabolismo energético e no mecanismo de fadiga muscular, algumas considerações tornam-se necessárias.

O ácido láctico é neutralizado no sangue por uma substância alcalina (o bicarbonato), presente no organismo, formando um sal (lactato), conforme a sequiência mostrada abaixo.

$\mathrm{C}_{3} \mathrm{H}_{6} \mathrm{O}_{3}$ (ácido láctico) $+\mathrm{NaHCO}_{3}$ (bicarbonato de sódio) $\rightarrow$ LACTATO de $\mathrm{Na}+\mathrm{H}_{2} \mathrm{CO}_{3}$ $\left(\mathrm{H}_{2} \mathrm{CO}_{3} \leftarrow \mathrm{H}_{2} \mathrm{O}+\mathrm{CO}_{2}\right) \Rightarrow$ LACTATO de $\mathrm{Na}+\mathrm{H}_{2} \mathrm{O}+\mathrm{CO}_{2}$

Esse processo de acúmulo ocasiona queda do $\mathrm{pH}$ sanguíneo ( $\mathrm{pH}$ do ácido láctico $~ 3,8 ; \mathrm{pH}$ celular $\sim 7$ ) e acelera o consumo de bicarbonato. Como vimos, a tentativa do organismo em tamponar esse ácido láctico em excesso vai resultar em lactato, água e gás carbônico. Este $\mathrm{CO}_{2}$ "extra", originado no tamponamento, vem juntar-se ao $\mathrm{CO}_{2}$ produzido pelo metabolismo aeróbio (via ciclo de Krebs e sistema de transporte de elétrons), o que vai provocar um desequilíbrio na relação $\mathrm{VCO}_{2} / \mathrm{VO}_{2}\left(\mathrm{o} \mathrm{VCO}_{2}\right.$ aumenta muito, comparado ao $\dot{\mathrm{V}}_{2}$ ). É quando observamos um aumento importante na "razão de trocas respiratórias" (R), ou seja: $\mathrm{R}=\mathrm{VCO}_{2} \uparrow \uparrow / \mathrm{VO}_{2} \uparrow \Rightarrow \mathrm{R} \uparrow$ ("aceleração de $\mathrm{R}$ ").

Isto ocorre porque, devido à acidose e ao excesso de $\mathrm{CO}_{2}$, ocorrerá a aceleração dos níveis de ventilação (hiperventilação) em relação ao consumo de oxigênio, com o gás carbônico "extra" podendo ser eliminado pelos pulmões (com "inflexão da curva de $\mathrm{VCO}_{2}$ "). A relação $\mathrm{VE} / \mathrm{V}_{2}$ (chamada equivalente ventilatório do oxigênio), que até este momento mantinha-se praticamente constante (por aumentos proporcionais de $\mathrm{VE}$ e $\mathrm{VO}_{2}$ ), passa a ter incrementos às custas do aumento maior de VE. É importante ressaltar que o maior fluxo ventilatório não consegue acelerar, na mesma proporção, o ritmo de consumo de $\mathrm{O}_{2}$. Assim, o crescimento de VE passa a ser superior ao do $\mathrm{VO}_{2}$, com conseqüente "perda da linearidade" de $\mathrm{VE} / \mathrm{VO}_{2}{ }^{28}$ (até o 1o limiar: $\mathrm{VE} \uparrow /$ $\dot{\mathrm{VO}}_{2} \uparrow \Rightarrow$ "constante"; acima do 1을 limiar: $\mathrm{VE} \uparrow \uparrow / \mathrm{VO}_{2} \uparrow \Rightarrow$ $\uparrow)$. Esse ponto é chamado de "primeiro limiar ventilatório" ou "limiar ventilatório I" (LVI).

A hiperventilação também provoca maiores valores de $\mathrm{PETO}_{2}$ (pressão expirada final - alveolar - de oxigênio) e da $\mathrm{FETO}_{2}$ (fração expirada final - alveolar - de oxigênio). Isto ocorre porque este aumento de fluxo ventilatório leva a um maior volume de oxigênio ofertado aos pulmões, mas que não é captado na mesma proporção. Esta "sobra" de oxigênio é "devolvida" no ar expirado, explicando o aumento na concentração de $\mathrm{O}_{2}$ na expiração e, portanto, de PETO $_{2}$ e FETO $_{2}$.

Acima do 1o limiar, o aumento da ventilação provoca uma aceleração no $\mathrm{CO}_{2}$ eliminado (o incremento na curva de VE tende a ser proporcional ao crescimento de $\mathrm{VCO}_{2}$ ), mantendo a relação $\mathrm{VE} / \mathrm{VCO}_{2}$ (equivalente ventilatório do $\mathrm{CO}_{2}$ ) próxima de uma constante. Este fenômeno é denominado "tamponamento isocápnico" 29.

Com a progressão dos níveis de intensidade de esforço, chega-se a um estágio onde ocorre acúmulo crescente e importante de ácido láctico, que poderíamos interpretar como sendo um estado de "esgotamento" dos mecanismos de tamponamento, o que vai ocasionar, novamente, importantes alterações nas curvas ventilatórias. É o chamado "segundo limiar ventilatório" ou "limiar ventilatório II" (LVII). Acima deste nível, haveria um acúmulo significativo de ácido láctico, com importantes repercussões no processo de fadiga muscular e conseqüente comprometimento da performance. Ultrapassado o 2 o limiar, na tentativa de compensar a acidose metabólica, o organismo provoca uma 
segunda aceleração na curva ventilatória (VE), mas desta vez não acompanhada de um aumento proporcional de $\mathrm{VCO}_{2}$ (estimulada pela acidose, a VE aumenta mais que a produção de $\mathrm{CO}_{2}$, cuja formação está comprometida pela menor eficiência do mecanismo de tamponamento nestes níveis de intensidade de carga ${ }^{28}$. Como resultado, ocorre perda de linearidade na relação $\mathrm{VE} / \mathrm{VCO}_{2}$, com aumento do equivalente ventilatório do $\mathrm{CO}_{2}$, à custa deste maior incremento de VE (até o 2o limiar: $\mathrm{VE} \uparrow / \mathrm{VCO}_{2} \uparrow \Rightarrow$ "constante"; acima do $2 \mathrm{o}$ limiar: $\left.\mathrm{VE} \uparrow \uparrow / \mathrm{VCO}_{2} \uparrow \Rightarrow \uparrow\right)$. Há também queda abrupta de PETCO $_{2}$ e FETCO $_{2}$ (o maior fluxo ventilatório, sem concomitante aumento proporcional de $\mathrm{CO}_{2}$, ocasiona menores concentrações deste gás no ar expirado).

É importante salientar que se pode encontrar na literatura referências sobre um limiar apenas (com citações sobre "o" limiar anaeróbio) que, na maioria das vezes, seria o 1 o limiar (denominado "limiar anaeróbio" por Wasserman e colaboradores). No entanto, existe ainda muita controvérsia sobre a terminologia a ser empregada, o que pode resultar em erros, principalmente quando comparam-se trabalhos alemães e escandinavos com os da escola americana. O "limiar anaeróbio", em estudos europeus, geralmente refere-se ao $2^{\circ}$ limiar, enquanto nos trabalhos americanos costuma-se utilizar o termo para o $1^{\circ}$ limiar $^{16}$.

Sabe-se que a curva de frequiência cardíaca, num teste de esforço progressivo, guarda estreita correlação com os níveis submáximos de $\mathrm{v}_{2}$. Por isso, os limiares podem ser expressos quanto às suas frequiências cardíacas (FCs) correspondentes. O uso prático destas FCs específicas (principalmente com o advento dos frequiencímetros) traz uma grande contribuição para a prescrição, observação e controle das atividades físicas e desportivas.

Mensuração e especificidade - na avaliação das capacidades físicas, seja em laboratório ou em testes de campo, o indicado é o uso de ergômetros e/ou testes que mais se aproximem das ações e gestos esportivos utilizados na modalidade praticada. As discrepâncias nos resultados de V $\mathrm{O}_{2}$ max e de limiares obtidos, para um mesmo indivíduo, quando utilizam-se ergômetros diferentes (bicicleta ergométrica e esteira, por exemplo) mostram que o respeito à especificidade dos testes promove os resultados mais próximos da realidade esportiva do avaliado.

Outra importante preocupação é a de que o teste aplicado tenha o devido embasamento científico, com bons níveis de confiabilidade e reprodutibilidade, além de um grau de precisão compatível com os objetivos da avaliação (no esporte de alto nível, especialmente, é injustificável que medidas e observações realizadas de modo empírico - ou quase - sirvam de parâmetro para prescrição de treinamento). Toda vez que, na falta de disponibilidade, seja ela financeira, estrutural ou tecnológica, venha a ser realizada uma avaliação mais "simples" (como, por exemplo, os testes de mensuração "indireta" de $\dot{v}_{2} \max$ ), deve-se conhecer, ao menos, as limitações e possibilidades de erro envolvidas na sua execução e coleta de dados, quando comparados aos exames com medida direta de gases.

A avaliação ergoespirométrica dos atletas paraolímpicos brasileiros, realizada no laboratório do CEFE-Unifesp, teve como objetivos principais a avaliação da capacidade funcional, com a mensuração do $\mathrm{V}_{2}$ max e dos limiares ventilatórios (LVI e LVII), e a pesquisa de eventuais fatores limitantes do desempenho.

\section{MÉTODOS}

Características especiais - para este grupo de atletas, com diferentes tipos e níveis de deficiências, algumas características especiais devem ser mencionadas.

Especificidade: foram utilizados ergômetros que melhor pudessem avaliar o gesto esportivo e estruturas orgânicas envolvidas em cada modalidade.

Segurança e cuidados especiais:

- Portadores de paralisia cerebral (PCs), por exemplo, precisam de cuidado redobrado, pela predisposição para a ocorrência de acidentes (quedas), quando da execução de testes na esteira rolante, por apresentarem um pior nível de coordenação neuromuscular, especialmente em velocidades mais altas. Quando se percebe que há condições cardiorrespiratórias para seguimento do teste, mas este pode vir a ser interrompido pelo avaliado por exacerbação da incoordenação, deve-se fazer os incrementos de carga às custas de inclinação e não de velocidade. Também para este tipo de deficiência, em especial, deve-se estar atento para aqueles que apresentam movimentação involuntária fascial ou angulação mandibular muito aguda, pois pode não ocorrer um adequado velamento nas máscaras ou nos bocais utilizados, com conseqüente "escape" na captação dos gases;

- Deficientes visuais necessitam manter, na maioria dos casos, algum contato das mãos com o corrimão da esteira (sem se apoiarem), além de poderem ter uma orientação verbal sobre sua biomecânica e situação espacial (em nossos testes, aqueles com maior dificuldade usaram "cintos" de segurança, presos à sua cintura e ao corrimão frontal da esteira).

Entendimento e motivação: é importante que os atletas sejam constantemente motivados para que se possa atingir o "real" esforço máximo. PCs e portadores de deficiência mental (DMs) são os que mais precisam de explicações prévias detalhadas sobre os testes, pela inerente dificuldade de entendimento. Uma alternativa eficaz, que geralmente ajuda no desempenho durante o teste é, quando possível, 
TABELA 1

Basquete masculino DM (esteira rolante)

\begin{tabular}{|c|c|c|c|c|c|c|}
\hline Nome & $\begin{array}{c}\dot{\mathrm{V}} \mathrm{O}_{2} \max \\
\left(\mathrm{mL} \cdot \mathrm{min}^{-1} \cdot \mathrm{kg}^{-1}\right)\end{array}$ & $\begin{array}{l}\dot{\mathrm{V}} \mathrm{O}_{2} \max \\
(\mathrm{L} / \mathrm{min})\end{array}$ & $\begin{array}{c}\dot{\mathrm{VO}} \mathrm{O}_{2}-\mathrm{LVI} \\
\left(\mathrm{mL} \cdot \mathrm{min}^{-1} \cdot \mathrm{kg}^{-1}\right)\end{array}$ & $\begin{array}{l}\text { FC-LVI } \\
\text { (bpm) }\end{array}$ & $\begin{array}{c}\dot{\text { VOO }} \mathrm{2}_{2} \text { LVII } \\
\left(\mathrm{mL} \cdot \mathrm{min}^{-1} \cdot \mathrm{kg}^{-1}\right)\end{array}$ & $\begin{array}{c}\text { FC-LVII } \\
\text { (bpm) }\end{array}$ \\
\hline R.C. & 45,9 & 3,35 & 32,7 & 163 & 36,9 & 176 \\
\hline N.C. & 41,0 & 4,08 & 26,0 & 157 & 33,8 & 170 \\
\hline L.G. & 51,1 & 4,57 & 32,4 & 151 & 41,3 & 168 \\
\hline J.S. & 50,5 & 4,24 & 32,1 & 159 & 40,2 & 177 \\
\hline I.B. & 52,0 & 4,98 & 34,6 & 169 & 43,6 & 184 \\
\hline G.S. & 61,8 & 3,39 & 40,9 & 176 & 48,2 & 194 \\
\hline D.C. & 56,8 & 3,72 & 33,7 & 163 & 43,0 & 180 \\
\hline C.S. & 52,7 & 3,83 & 31,0 & 158 & 42,3 & 174 \\
\hline A.Sa. & 40,6 & 3,17 & 24,4 & 146 & 32,7 & 166 \\
\hline A.C. & 46,5 & 3,17 & 32,7 & 158 & 39,6 & 175 \\
\hline A.L. & 41,8 & 2,99 & 23,0 & 155 & 33,7 & 174 \\
\hline A.So. & 55,7 & 3,66 & 39,2 & 178 & 47,0 & 193 \\
\hline Média & 49,7 & 3,76 & 31,8 & & 40,2 & \\
\hline D.P. & 6,7 & 0,61 & 5,6 & & 5,3 & \\
\hline
\end{tabular}

Valores de referência ${ }^{30}: \dot{V}_{2}$ max relativo $=39,6 ;$ absoluto $=2,8$.

TABELA 2

Futebol masculino PC (esteira rolante)

\begin{tabular}{|c|c|c|c|c|c|c|}
\hline Nome & $\begin{array}{c}\dot{\mathrm{V}} \mathrm{O}_{2} \max \\
\left(\mathrm{mL} \cdot \mathrm{min}^{-1} \cdot \mathrm{kg}^{-1}\right)\end{array}$ & $\begin{array}{l}\dot{\mathrm{V}} \mathrm{O}_{2} \max \\
\text { (L/min) }\end{array}$ & $\begin{array}{c}\dot{\mathrm{V}} \mathrm{O}_{2}-\mathrm{LVI} \\
\left(\mathrm{mL} \cdot \mathrm{min}^{-1} \cdot \mathbf{k g}^{-1}\right)\end{array}$ & $\begin{array}{l}\text { FC-LVI } \\
\text { (bpm) }\end{array}$ & $\begin{array}{c}\dot{\text { VOO }} \mathrm{L}_{2} \text {-LVII } \\
\left(\mathrm{mL} \cdot \mathrm{min}^{-1} \cdot \mathrm{kg}^{-1}\right)\end{array}$ & $\begin{array}{c}\text { FC-LVII } \\
\text { (bpm) }\end{array}$ \\
\hline A.C. & 45,6 & 2,89 & 29,0 & 162 & 37,1 & 183 \\
\hline D.A. & 46,9 & 3,58 & 29,7 & 145 & 35,9 & 164 \\
\hline F.F. & 49,8 & 3,13 & 32,4 & 143 & 42,5 & 162 \\
\hline J.F. & 50,7 & 3,70 & 34,9 & 158 & 43,7 & 172 \\
\hline L.R. & 51,1 & 3,67 & 37,8 & 171 & 43,8 & 182 \\
\hline M.L. & 48,2 & 3,05 & 33,2 & 167 & 39,8 & 175 \\
\hline M.W. & 55,7 & 3,91 & 37,5 & 168 & 45,6 & 184 \\
\hline M.Sa. & 47,2 & 3,29 & 32,3 & 173 & 40,4 & 190 \\
\hline M.Si. & 57,7 & 3,70 & 34,2 & 168 & 46,6 & 186 \\
\hline J.R. & 55,8 & 3,40 & 31,9 & 163 & 42,9 & 188 \\
\hline Média & 50,9 & 3,43 & 33,3 & & 41,8 & \\
\hline D.P. & 4,2 & 0,33 & 2,9 & & 3,5 & \\
\hline
\end{tabular}

Valores de referência $*$ : $\mathrm{O}_{2}$ max relativo $=50,6$; absoluto $=3,3$.

* dados de avaliações no CEFE-Unifesp, mesma modalidade, para "Atlanta 96".

TABELA 3

Atletismo masculino DV (esteira rolante)

\begin{tabular}{|c|c|c|c|c|c|c|}
\hline Nome & $\begin{array}{c}\dot{\mathrm{V}} \mathrm{O}_{2} \max \\
\left(\mathrm{mL} \cdot \mathrm{min}^{-1} \cdot \mathbf{k g}^{-1}\right)\end{array}$ & $\begin{array}{l}\dot{\mathrm{V}} \mathrm{O}_{2} \max \\
\text { (L/min) }\end{array}$ & $\begin{array}{c}\dot{\mathrm{V}} \mathrm{O}_{2}-\mathrm{LVI} \\
\left(\mathrm{mL} \cdot \mathrm{min}^{-1} \cdot \mathrm{kg}^{-1}\right)\end{array}$ & $\begin{array}{l}\text { FC-LVI } \\
\text { (bpm) }\end{array}$ & $\begin{array}{c}\dot{\text { VO }} \mathrm{2}_{2} \text { LVII } \\
\left(\mathrm{mL} \cdot \mathrm{min}^{-1} \cdot \mathbf{k g}^{-1}\right)\end{array}$ & $\begin{array}{r}\text { FC-LVII } \\
\text { (bpm) }\end{array}$ \\
\hline A.A. & 61,1 & 3,73 & 37,8 & 167 & 49,7 & 188 \\
\hline A.As. & 63,5 & 4,05 & 40,3 & 157 & 55,3 & 175 \\
\hline A.So. & 64,2 & 4,39 & 38,4 & 159 & 48,3 & 178 \\
\hline Média & 62,9 & 4,06 & 38,8 & & 51,1 & \\
\hline D.P. & 1,6 & 0,33 & 1,3 & & 3,7 & \\
\hline
\end{tabular}

Valores de referência* $\dot{\mathrm{VO}}_{2}$ max relativo $=57,0$; absoluto $=3,7$.

* dados de avaliações no CEFE-Unifesp, mesma modalidade, para "Atlanta 96". 
TABELA 4

Atletismo masculino DM (esteira rolante)

\begin{tabular}{|c|c|c|c|c|c|c|}
\hline Nome & $\begin{array}{c}\dot{\mathrm{V}} \mathrm{O}_{2} \max \\
\left(\mathrm{mL} \cdot \mathrm{min}^{-1} \cdot \mathrm{kg}^{-1}\right)\end{array}$ & $\begin{array}{l}\dot{\mathrm{V}} \mathrm{O}_{2} \max \\
\text { (L/min) }\end{array}$ & $\begin{array}{c}\dot{\mathrm{V}} \mathrm{O}_{2}-\mathrm{LVI} \\
\left(\mathrm{mL} \cdot \mathrm{min}^{-1} \cdot \mathrm{kg}^{-1}\right)\end{array}$ & $\begin{array}{l}\text { FC-LVI } \\
\text { (bpm) }\end{array}$ & $\begin{array}{c}\dot{\text { VO }} \mathrm{2}_{2}-\mathrm{LVII} \\
\left(\mathrm{mL} \cdot \mathrm{min}^{-1} \cdot \mathrm{kg}^{-1}\right)\end{array}$ & $\begin{array}{r}\text { FC-LVII } \\
\text { (bpm) }\end{array}$ \\
\hline S.S. & 51,5 & 3,69 & 34,8 & 176 & 41,9 & 187 \\
\hline
\end{tabular}

TABELA 5

Atletismo feminino DV (esteira rolante)

\begin{tabular}{|c|c|c|c|c|c|c|}
\hline Nome & $\begin{array}{c}\dot{\mathrm{V}} \mathrm{O}_{2} \max \\
\left(\mathrm{mL} \cdot \mathrm{min}^{-1} \cdot \mathrm{kg}^{-1}\right)\end{array}$ & $\begin{array}{l}\dot{\mathrm{V}} \mathrm{O}_{2} \max \\
(\mathrm{L} / \mathrm{min})\end{array}$ & $\begin{array}{c}\dot{\mathrm{V}} \mathrm{O}_{2}-\mathrm{LVI} \\
\left(\mathrm{mL} \cdot \mathrm{min}^{-1} \cdot \mathrm{kg}^{-1}\right)\end{array}$ & $\begin{array}{l}\text { FC-LVI } \\
\text { (bpm) }\end{array}$ & $\begin{array}{c}\dot{\text { VOO }} \mathrm{2}_{2} \text { LVII } \\
\left(\mathrm{mL} \cdot \mathrm{min}^{-1} \cdot \mathrm{kg}^{-1}\right)\end{array}$ & $\begin{array}{r}\text { FC-LVII } \\
\text { (bpm) }\end{array}$ \\
\hline A.S. & 42,4 & 2,22 & 32,4 & 161 & 37,7 & 178 \\
\hline M.F. & 48,7 & 2,70 & 29,0 & 167 & 39,1 & 183 \\
\hline Média & 45,6 & 2,46 & 30,7 & & 38,4 & \\
\hline D.P. & 4,5 & 0,34 & 2,4 & & 1,0 & \\
\hline
\end{tabular}

\begin{tabular}{|c|c|c|c|c|c|c|}
\hline \multicolumn{7}{|c|}{$\begin{array}{c}\text { TABELA } 6 \\
\text { Judô masculino DV (esteira rolante) }\end{array}$} \\
\hline Nome & $\begin{array}{c}\dot{\mathrm{VO}} \mathrm{m}_{2} \max \\
\left(\mathrm{mL} \cdot \mathrm{min}^{-1} \cdot \mathbf{k g}^{-1}\right)\end{array}$ & $\begin{array}{l}\dot{\mathrm{VO}} \mathrm{O}_{2} \max \\
\text { (L/min) }\end{array}$ & $\begin{array}{c}\dot{\mathrm{VO}} \mathrm{O}_{2}-\mathrm{LVI} \\
\left(\mathrm{mL} \cdot \mathrm{min}^{-1} \cdot \mathrm{kg}^{-1}\right)\end{array}$ & $\begin{array}{l}\text { FC-LVI } \\
\text { (bpm) }\end{array}$ & $\begin{array}{c}\dot{\mathrm{VO}} \mathrm{O}_{2}-\mathrm{LVII} \\
\left(\mathrm{mL} \cdot \mathrm{min}^{-1} \cdot \mathrm{kg}^{-1}\right)\end{array}$ & $\begin{array}{l}\text { FC-LVII } \\
\text { (bpm) }\end{array}$ \\
\hline A.T. & 52,0 & 4,94 & 33,2 & 156 & 41,4 & 174 \\
\hline D.D. & 37,1 & 3,67 & 22,5 & 156 & 30,5 & 168 \\
\hline H.A & 46,4 & 2,92 & 29,9 & 158 & 37,7 & 178 \\
\hline L.F. & 29,3 & 3,33 & 20,8 & 148 & 27,4 & 166 \\
\hline Média & 41,2 & 3,72 & 26,6 & & 34,3 & \\
\hline D.P. & 10,0 & 0,87 & 5,9 & & 6,4 & \\
\hline
\end{tabular}

Valores de referência $*$ : $\mathrm{VO}_{2}$ max relativo $=45,5$; absoluto $=3,6$.

* dados de avaliações no CEFE-Unifesp, mesma modalidade, para "Atlanta 96".

\begin{tabular}{lcccccc}
\hline \multicolumn{7}{c}{ TABELA 7 } \\
Ciclismo masculino AMP (bicicleta ergométrica) \\
\hline
\end{tabular}


realizar os primeiros exames do dia com atletas já avaliados em outras oportunidades (mais "experientes"), fazendo com que os "novatos" possam assistir e incorporar melhor o modo de execução e os objetivos do teste.

\section{Protocolos}

Esteira rolante - início (aquecimento) $=5 \mathrm{~km} / \mathrm{h}$, durante 5 minutos; após, incremento de carga de $1 \mathrm{~km} / \mathrm{h}$ a cada minuto; final $=$ exaustão $($ com inclinação de $2,5 \%$ por minuto em cargas mais altas, caso necessário). Para os "guias" das corredoras deficientes visuais, a carga inicial foi de 10$12 \mathrm{~km} / \mathrm{h}$, com os incrementos de carga semelhantes aos dos atletas. Na avaliação dos atletas de "corrida", especificamente, a esteira partiu com inclinação inicial de $1 \%$.

Bicicleta - na bicicleta ergométrica, início (aquecimento) $=100 \mathrm{watts}$, por 5 minutos; após, aumento de $25 \mathrm{w} / \mathrm{mi}-$ nuto, até a exaustão. Também foi feita uma avaliação com os ciclistas usando seu próprio equipamento, acoplado a um sistema de rolamento para a roda traseira ("rolo"): iní- cio $($ aquecimento $)=$ velocidade de $30 \mathrm{~km} / \mathrm{h}$, durante $5 \mathrm{mi}-$ nutos; após, incremento de $3 \mathrm{~km} / \mathrm{h}$ a cada minuto. Este sistema mostrou-se ineficaz para a obtenção de esforço máximo, pois o aumento de carga exclusivamente às custas de velocidade não permitiu que isto ocorresse (teste interrompido principalmente por incoordenação neuromuscular).

Ergômetro de braços - início (aquecimento) $=25$ watts, durante 2 minutos; aumentos de $12,5 \mathrm{w}$ a cada minuto, até a exaustão. Não foi possível utilizar os resultados do teste de um atleta (halterofilismo), portador de uma deficiência que comprometia bastante a preensão da mão e a coordenação de um dos braços, o que inviabilizou a correta execução do teste.

\section{RESULTADOS}

É importante lembrar que estes dados devem ser avaliados levando-se em conta todas as ressalvas e detalhes discorridos anteriormente, principalmente acerca dos diversos fatores (sexo, tipo de ergômetro, etc.) que influenciam

\begin{tabular}{|c|c|c|c|c|c|c|}
\hline \multicolumn{7}{|c|}{$\begin{array}{c}\text { TABELA } 8 \\
\text { Halterofilismo masculino PM (ergômetro de braços) }\end{array}$} \\
\hline Nome & $\begin{array}{c}\dot{\mathrm{VO}} \mathrm{O}_{2} \max \\
\left(\mathrm{mL} \cdot \mathrm{min}^{-1} \cdot \mathbf{k g}^{-1}\right)\end{array}$ & $\begin{array}{l}\dot{\mathrm{VO}}_{2} \max \\
(\mathrm{L} / \mathrm{min})\end{array}$ & $\begin{array}{c}\dot{\mathrm{VO}} \mathrm{2}_{2}^{-\mathrm{LVI}} \\
\left(\mathrm{mL} \cdot \mathrm{min}^{-1} \cdot \mathbf{k g}^{-1}\right)\end{array}$ & $\begin{array}{l}\text { FC-LVI } \\
\text { (bpm) }\end{array}$ & $\begin{array}{c}\dot{\mathrm{VO}}{ }_{2}^{-L V I I} \\
\left(\mathrm{~mL} \cdot \mathrm{min}^{-1} \cdot \mathbf{k g}^{-1}\right)\end{array}$ & $\begin{array}{l}\text { FC-LVI } \\
\text { (bpm) }\end{array}$ \\
\hline J.B. & 33,5 & 2,41 & 16,7 & 135 & 21,0 & 149 \\
\hline
\end{tabular}

TABELA 9

Halterofilismo feminino AMP (ergômetro de braços)

\begin{tabular}{|c|c|c|c|c|c|c|}
\hline Nome & $\begin{array}{c}\dot{\mathrm{VO}} \mathrm{O}_{2} \max \\
\left(\mathrm{mL} \cdot \mathrm{min}^{-1} \cdot \mathbf{k g}^{-1}\right)\end{array}$ & $\begin{array}{l}\dot{\mathrm{V}} \mathrm{O}_{2} \max \\
(\mathrm{L} / \mathrm{min})\end{array}$ & $\begin{array}{c}\dot{\mathrm{VO}}{ }_{2}-\mathrm{LVI} \\
\left(\mathrm{mL} \cdot \mathrm{min}^{-1} \cdot \mathbf{k g}^{-1}\right)\end{array}$ & $\begin{array}{l}\text { FC-LVI } \\
\text { (bpm) }\end{array}$ & $\begin{array}{c}\dot{\mathrm{V} \mathrm{O}_{2}-\mathrm{LVII}} \\
\left(\mathrm{mL} \cdot \mathrm{min}^{-1} \cdot \mathrm{kg}^{-1}\right)\end{array}$ & $\begin{array}{l}\text { FC-LVII } \\
\text { (bpm) }\end{array}$ \\
\hline T.S. & 20,1 & 1,12 & 9,8 & 143 & 12,0 & 163 \\
\hline
\end{tabular}

TABELA 10

Valores médios ( ${ }^{\circ} \mathrm{O}_{2}$ max e $\dot{\mathrm{VO}}_{2}$ dos limiares) por modalidade

\begin{tabular}{|c|c|c|c|}
\hline $\begin{array}{c}\text { Modalidade } \\
\text { ( } \mathrm{n}=\text { número de atletas) }\end{array}$ & $\begin{array}{c}\dot{\mathrm{V}} \mathrm{O}_{2} \max \\
\left(\mathrm{mL} \cdot \mathrm{min}^{-1} \cdot \mathrm{kg}^{-1}\right)\end{array}$ & $\begin{array}{c}\dot{\mathrm{V}} \mathrm{O}_{2}-\mathrm{LVI} \\
\left(\mathrm{mL} \cdot \mathrm{min}^{-1} \cdot \mathrm{kg}^{-1}\right)\end{array}$ & $\begin{array}{c}\dot{\mathrm{VO}} \mathrm{2}_{2}-\mathrm{LVII} \\
\left(\mathrm{mL} \cdot \mathrm{min}^{-1} \cdot \mathbf{k g}^{-1}\right)\end{array}$ \\
\hline Ciclismo masc. (2) & 63,7 & 41,1 & 51,5 \\
\hline Atletismo masc. (4) & 60,1 & 37,8 & 48,8 \\
\hline Futebol (10) & 50,9 & 33,3 & 41,8 \\
\hline Basquete masc. (12) & 49,7 & 31,8 & 40,2 \\
\hline Atletismo fem. (2) & 45,6 & 30,7 & 38,4 \\
\hline Judô masc. (4) & 41,2 & 26,2 & 34,3 \\
\hline Halterofilismo masc. (1) & 30,9 & 19,3 & 22,8 \\
\hline Halterofilismo fem. (1) & 20,4 & 9,9 & 12,0 \\
\hline
\end{tabular}


a mensuração dos parâmetros ergoespirométricos. Apesar de, em alguns casos, ser mais adequado o uso do termo "pico de $\dot{\mathrm{O}}_{2}$ ", conforme discutido anteriormente, a denominação " $\mathrm{VO}_{2}$ max" foi padronizada para todos os testes executados.

Apresentamos, a seguir, os principais resultados dos atletas paraolímpicos, sexo masculino, das modalidades de atletismo - "corrida" (deficiência visual - DV; deficiência mental - DM), basquete (DM), ciclismo (amputação - AMP), futebol (paralisia cerebral - PC), halterofilismo (poliomielite - PM) e judô (DV); sexo feminino, atletas do atletismo - "corrida" (DV) e halterofilismo (AMP).

A atleta Adria Santos (A.S.), medalhista (ouro e prata) em Sidney nos 100 e 200 metros, não teve resultados expressivos neste teste, o que é explicável pela própria característica de predomínio de velocidade em suas provas.

$\mathrm{O}$ atleta Antonio Tenório (A.T.), que foi medalha de ouro em Sidney, apresentou resultados superiores ao restante do grupo, mostrando ter um bom condicionamento físico. Seu esporte exige ações específicas "explosivas", mas a presença de uma boa aptidão cardiorrespiratória pode ajudar na manutenção do desempenho durante a seqüência de lutas, além de proporcionar uma adequada recuperação.

Os maiores valores de $\dot{\mathrm{VO}}_{2}$ (relativo e absoluto) obtidos no "rolo" (R.S. = 50,9 e 3,55; C.S. = 49,1 e 3,03), bem inferiores aos obtidos na ergométrica, mostraram a ineficácia deste sistema em fazer estes atletas atingirem o esforço máximo.

Não foi possível utilizar os resultados de um halterofilista, que apresentou problemas físicos no dia de realização do seu teste, inviabilizando a correta execução do exame.

\section{DISCUSSÃO}

É interessante notar que, como esperado, as modalidades com característica predominante de resistência são as que mostram os melhores resultados (no grupo de atletismo masculino há, inclusive, maratonistas). Características como sexo e ergômetro utilizado devem ser consideradas, com os menores valores sendo obtidos em ergômetro de braços e sexo feminino.

Ainda hoje, há pouca disponibilidade de estudos e dados publicados na literatura, envolvendo atletas paraolímpicos, mesmo em países mais desenvolvidos. Isto faz com que a experiência adquirida por nosso laboratório, ao longo dos anos, especialmente nos trabalhos em associação com o Comitê Paraolímpico Brasileiro, possa colaborar para o incremento da pesquisa sobre os diversos tipos de deficiências e, assim, implementar ações práticas, não apenas para a esfera competitiva, mas também para o grande con- tingente de portadores de necessidades especiais que temos no país. Quanto maior o conhecimento sobre este assunto, mais barreiras vão sendo vencidas, refletindo-se na integração cada vez mais efetiva destes indivíduos em suas comunidades, além de servir de orientação e incentivo para o surgimento de várias alternativas de programas específicos, visando a melhora da saúde e da qualidade de vida desta população.

\section{AGRADECIMENTOS}

- Comitê Paraolímpico Brasileiro (CPB)

- Secretaria Nacional de Esportes

- Rede Cenesp/Unifesp

- Associação Fundo de Incentivo à Psicofarmacologia (Afip)

- Universidade Federal de São Paulo (Unifesp)

\section{REFERÊNCIAS}

1. Astrand PO, Rodahl K. Textbook of work physiology. New York: McGraw-Hill, 1986

2. Wasserman K, Whipp BJ. Exercise physiology in health and disease. Am Rev Respir Dis 1975;112:219.

3. Taylor HL, Buskirk E, Henschel A. Maximal oxygen uptake as an objective measure of cardiorespiratory performance. J Appl Physiol 1955;8:73.

4. Rowland, TW. Does peak $\dot{\mathrm{VO}}_{2}$ reflect $\dot{\mathrm{VO}}_{2} \max$ in children? Med Sci Sports Exerc 1993;25:689.

5. Wasserman K, Hansen JE, Sue DY, Whipp BJ. Principles of exercise testing and interpretation. Philadelphia: Lea \& Febiger, 1987.

6. Pollock ML, Foster C, Rod JL, Wible G. Comparison of methods for determining exercise training intensity for cardiac patients and healthy adults. In: Kellerman JJ, editor. Comprehensive cardiac rehabilitation. Basel: S. Karger, 1982:129-33.

7. McMiken D, Daniels J. Aerobic requirements of maximal aerobic power in treadmill and track running. Med Sci Sports Exerc 1976;8:14-7.

8. Williams JS, Powers S, Stuart S. Hemoglobin desaturation in highly trained athletes during heavy exercise. Med Sci Sports Exerc 1986;18: 168-73.

9. Vogel JA, Patton JF, Mello RP, Daniels WL. An analysis of aerobic capacity in a large United States population. J Appl Physiol 1986;60:494500.

10. Bergh U. The influence of body mass in cross-country skiing. Med Sci Sports Exerc 1987;19:324-31.

11. Kohrt WM, O'Connor JS, Skinner JS. Longitudinal assessment of responses by triathletes to swimming, cycling, and running. Med Sci Sports Exerc 1989;21:569-75.

12. Miles DS, et al. Cardiovascular responses to upper body exercise in normal and cardiac patients. Med Sci Sports Exerc 1989;21:S126.

13. Sawka M, et al. Determination of maximal of aerobic power during upper body exercise. J Appl Physiol 1983;54:113-7.

14. Bouchard C, Dione FT, Simoneau JA, Boulay MR. Genetics of aerobic and anaerobic performances. Exerc Sports Sci Rev 1992;20:27-58.

15. Wasserman K. The chairman's postconference reflections. Am Rev Respir Dis 1984;129:S1-S2.

16. Ribeiro JP, Hughes V, Fielding RA, Holden W, Evans W, Knuttgen HG. Metabolic and ventilatory responses to steady state exercise relative to lactate thresholds. Eur J Appl Physiol 1986;55:215-21. 
17. Rusko H, Luhtanen P, Rahkila P, Viitasalo J, Rehunen S, Harkonen M. Muscle metabolism, blood lactate and oxygen uptake in steady state exercise at aerobic and anaerobic thresholds. Eur J Appl Physiol 1986; 55:181-6.

18. Donovan CM, Brooks GA. Endurance training affects lactate clearance, not lactate production. Am J Physiol 1983;244:E83-92.

19. Mazzeo RS, Brooks GA, Schoeller DA, Budinger TF. Disposal of blood [1-13C] lactate in humans during rest and exercise. J Appl Physiol 1986; 60:232-41.

20. Gollnick P, Hermansen L. Biochemical adaptations to exercise - anaerobic metabolism. In: Wilmore J, editor. Exercise and sport reviews. New York: Academic Pres, 1973.

21. Wasserman K, Beaver WL, Whipp BJ. Mechanisms and patterns of blood lactate increase during exercise in man. Med Sci Sports Exerc 1986;3: $344-52$.

22. Wasserman K. The peripheral circulation and lactic acid metabolism in heart, or cardiovascular, failure. Circulation 1989;80:1084-6.

23. Gaesser GA, Brooks G. Metabolic bases of the excess post-exercise oxygen consumption: a review. Med Sci Sports Exerc 1984;16:29-43.
24. Gollnick P, Bayly, Hodgston DR. Exercise intensity, training, diet, and lactate concentration in muscle and blood. Med Sci Sports Exerc 1986; $18: 334-40$

25. Connett RJ, Gayeski TE, Honing CR. Lactate efflux in unrelated to intracellular $\mathrm{PO}_{2}$ in working red muscle in situ. J Appl Physiol 1986;61: 402-8.

26. Stryer L. Biochemistry. San Francisco: W.H. Freeman, 1988

27. Wasserman K, Whipp BJ, Koyal SN, Beaver WL. Anaerobic threshold and respiratory gas exchange during exercise. J Appl Physiol 1973;35: 236-43.

28. Wasserman K. The anaerobic threshold measurement to evaluate exercise performance. Am Rev Dis 1984;129:S35-S40.

29. Wasserman K, Whipp BJ, Casaburi R, Beaver WL, Brown HV. $\mathrm{CO}_{2}$ flow to the lungs and ventilatory control. In: Dempsey JA, Reed CE, editors. Muscular exercise and the lung. Madison, University of Wisconsin Press, 1977:103-35.

30. Montgomery DL, et al. The effects of two physical fitness program designed for mentally retarded adults. Can J Sports Sci 1988;13:73-8. 